\title{
Fatores de risco cardiovascular - Perfil clínico e epidemiológico dos participantes do projeto Atividade Física na Vila
}

\author{
Cardiovascular Risk Factors - Clinical and Epidemiologic \\ profiles in the subjects of the "Atividade Física na Vila" Project
}

Eduardo Simon* Tatiana Tavares Silva** José Silvio O. Barbosa***

Resumo Ricardo Donato Rodrigues**** Rosimere de Jesus Teixeira*****

As doenças cardiovasculares aterotrombóticas representam uma das principais causas de morte no mundo. A aterosclerose é uma patologia de origem multifatorial com grande dependência genética e familiar, que apresenta elevada suscetibilidade de agravamento, segundo o estilo de vida. Nosso objetivo foi descrever as prevalências dos fatores de risco cardiovascular (RCV) e da Síndrome Metabólica (SM), além de estimar o RCV nos participantes do projeto Atividade Física na Vila. Mediante estudo de corte seccional, 48 pacientes entre 23 e 74 anos (41 mulheres e 7 homens), foram avaliados quanto à presença de sedentarismo, tabagismo, história familiar de diabetes mellitus tipo 2 (DM2) ou doença arterial coronariana, obesidade, obesidade abdominal, hipertensão arterial (HA), DM2, glicemia de jejum alterada (GjA), dislipidemia e SM. As prevalências dos fatores de risco foram comparadas com os dados disponíveis para a população geral. A SM foi definida pelos critérios da NCEP. O RCV foi estimado pelo Escore de Framingham (EF) e comparado entre os participantes com e sem SM. Dos pacientes, $67 \%$ tinham mais de 50 anos e 46\%, mais de 55 . Houve alta prevalência dos fatores de RCV comparando-se aos dados populacionais, destacando-se sedentarismo ( $52 \% \times 56 \%$; NS), obesidade (homens 14\% x 9\%; NS; mulheres 39\% x 13\%; p <0,05), HA ( $56 \%$ x 32\%; $<<0,05)$ e hipercolesterolemia $(63 \%$ x 40\%; $<<0,05)$. As prevalências de DM, GjA, obesidade abdominal e SM foram de 10\%, 21\%, $60 \%$ e 27\%, respectivamente. O EF foi de 5,2 \pm 5,5, sendo significativamente maior nos pacientes com SM $(9,5 \pm 3,1 \times 3,5 \pm 5,4 ; \mathrm{p}<0,05)$. Esses dados evidenciam a alta prevalência dos fatores de risco e sugerem sua ação sinérgica no aumento do RCV global representado pelo EF e a necessidade das mudanças no estilo de vida desses pacientes.

\section{Abstract}

The group of atherothrombotic cardiovascular diseases represents one of the leading causes of death around the world. Atherosclerosis is a condition of multifactorial origin, with great genetic and familiar dependence, besides being characterized by an elevated aggravation trend related to lifestyle. Our objective was to describe the prevalence of cardiovascular risk. (CVR) factors and Metabolic Syndrome (MS), as well

Palavras-chave: Doenças Cardiovasculares;

Perfil de Saúde; Atividade Física.
Key Words: Cardiovascular Diseases; Health Profile;

Motor Activity.

*Residente de Medicina Familiar e Comunitária do Departamento de Medicina Integral, Familiar e Comunitária. Universidade do Estado Rio de Janeiro.

**Doutoranda em Medicina/UERJ, professora assistente do Departamento de Medicina Integral, Familiar e Comunitária, Universidade do Estado Rio de Janeiro.

***Doutorando em Medicina/UERJ, professor assistente do Laboratório de Fisiologia Aplicada de Educação Física, Universidade do Estado Rio de Janeiro.

****Doutor em Saúde Coletiva, professor adjunto do Departamento de Medicina Integral, Familiar e Comunitária, Universidade do Estado Rio de Janeiro.

*****DDoutora em Endocrinologia, professora. visitante do DMIFC, Universidade do Estado Rio de Janeiro. 
as to estimate the cardiovascular risk in the subjects of the "Atividade Fisica na Vila" Project. By means of a cross sectional study, 48 patients aged between 23 and 74 (41 women and 7 men) were screened for the presence of sedentary lifestyle, smoking, family antecedents of Diabetes mellitus type 22 (DM) or Coronary artery disease, obesity, abdominal obesity, High Blood Pressure (HBP), DM2, Impaired Fasting Glucose (IFG), Lipid abnormalities, and MS. This prevalence was compared to the available general population data. The MS definition was that of the NCEP. The CV $\mathrm{R}$ was estimated using the Framingham Score (FS), and compared among the groups with and without MS. $67 \%$ of the patients were 50 or older and $46 \%$ were 55 or older. There was a high prevalence of $C V R$ factors compared to the population data, specially in the case of sedentary lifestyle (52\% × 56\%, NS), obesity (men 14\% × 9\%, NS; women $39 \% \times 13 \%, P<0.05), \mathrm{HBP}(56 \% \times 32 \%, P<0.05)$, high total cholesterol $(63 \% \times 40 \%, P<0.05)$. The prevalence of DM2, IFG, abdominal obesity and MS was 10\%, 21\%, 60\% and 27\%, respectively. The mean FS was 5,2 $\pm 5,5$, significantly higher in the patients with $M S(9,5 \pm 3,1 \times 3,5 \pm 5,4 p<0.05)$. These data indicate the high prevalence of $C V \mathrm{R}$ factors, and suggest its synergy in elevating the global CV $\mathrm{R}$ represented by the FS, as well as strongly support the need for changes in the lifestyle of these patients.

\section{Introdução}

A doença cardiovascular aterosclerótica é, em termos proporcionais, a principal causa de mortalidade em países ditos desenvolvidos e em muitos países em desenvolvimento, como o Brasil. Da mesma forma, o impacto deste grupo de doenças nos gastos com saúde é significativo, havendo inclusive uma tendência ascendente, visto que está ocorrendo o aumento da prevalência global de alguns dos seus principais fatores de risco ${ }^{1}$. A epidemia de obesidade e doenças associadas como diabetes mellitus tipo 2 (DM2), hipertensão arterial (HA) e dislipidemia representam um dos maiores problemas de saúde pública da atualidade ${ }^{2}$. O que fazer para controlar esse problema?

Na primeira metade do século 20, experimentos com animais e observações clínicas ligaram certas condições, como a hipercolesterolemia, ao risco aumentado de eventos aterotrombóticos ${ }^{3}$. A partir daí, principalmente após a avaliação dos desfechos da clássica coorte de Framingham, juntamente com outros estudos prospectivos, está bem estabelecida na literatura a relação entre alguns fatores e o risco de doenças cardiovasculares (DCV) ${ }^{4,5}$. A lista dos chamados fatores de risco cardiovascular (RCV) continua crescendo, dentre estes já implicados na patogênese da aterosclerose, podemos listar: tabagismo, DM2, dislipidemia, história familiar de infarto precoce do miocárdio, resistência periférica à insulina $(\mathrm{RI})$, obesidade e sedentarismo. Outros fatores, como níveis séricos aumentados de homocisteína, lipoproteína (a), fibrinogênio e proteína $C$ reativa, bem como algumas infecções crônicas por organismos como espécies do gênero Chlamydia vêm sendo investigados quanto a suas possíveis implicações neste processo ${ }^{3}$.

Mais recentemente, tem-se disseminado o entendimento de que tais fatores têm uma etiopatogênese comum. Segundo este entendimento, um hábito de vida sedentário, um ambiente ansiogênico e uma alimentação hipercalórica rica em carboidratos e gorduras saturadas levariam, ao longo do tempo, à obesidade, ao aumento gradual da RI, à dislipidemia, à HA, e à DM2, dentre outros problemas ${ }^{1}$. O conceito de Síndrome Metabólica (SM) - anteriormente chamada de síndrome X, dismetabólica ou plurimetabólica - representado por um conjunto de fatores de risco tanto para o DM2 como para a DCV, tendo a RI como fio condutor etiopatogênico, veio juntar-se a esta discussão. A SM apresentase como a manifestação precoce do solo comum que leva à DCV, sendo sua presença um preditor importante de morbimortalidade $e^{6,7,8,9}$.

A citada clareza e precisão na associação entre determinadas condições e o risco de doença arterial coronariana (DAC) é tal, que têm sido desenvolvidas funções matemáticas para estimar o risco do desenvolvimento desta num dado período de tempo, a partir da presença de um maior ou menor número dos citados fatores. Dentre tais funções, destaca-se o Escore de Framingham, que deve seu nome à já citada coorte norte-americana com base na qual foi construído. Este escore é adotado para estimar a probabilidade de ocorrer infarto do miocárdio ou morte 
por doença coronária em curto prazo, isto é, no período de dez anos, em indivíduos sem diagnóstico prévio de aterosclerose clínica 4 .

O projeto Atividade Física na Vila é voltado para pacientes do programa Saúde na Vila ou para pacientes do Ambulatório de Medicina Integral (AMI) do Hospital Universitário Pedro Ernesto (HUPE) que moram nas adjacências do prédio. Este projeto visa promover a educação para a saúde, o auto-cuidado e a ampliação da rede social, utilizando a atividade física como prática integradora, bem como fornecer subsídios para o estudo de seus efeitos sobre a saúde. O objetivo deste trabalho foi descrever a prevalência dos principais fatores de RCV e da SM assim como determinar o RCV nos participantes do projeto Atividade Física na Vila. Não devemos esquecer que o reconhecimento e o conseqüente controle dos fatores de risco dessa enfermidade são básicos na prevenção da doença cardiovascular.

\section{Casuística e Métodos}

O estudo, do tipo observacional transversal, foi desenvolvido no ambulatório do projeto Atividade Física na Vila, no HUPE / UERJ, e teve por população os 106 pacientes envolvidos no projeto. O Atividade Física na Vila é uma parceria entre o Departamento de Medicina Integral, Familiar e Comunitária (DMIFC) e o Laboratório de Fisiologia Aplicada de Educação Física, e inclui participantes de ambos os gêneros, entre 20 e 75 anos, adscritos no programa Saúde na Vila, do DMIFC, ou oriundos do AMI ainda não adscritos no programa, bem como de outros ambulatórios do HUPE, mas que desejam realizar atividade física supervisionada. Após avaliação clínica e laboratorial, os pacientes são liberados, com ou sem restrições, à prática supervisionada de exercícios físicos ou encaminhados à investigação clínica adicional ou tratamento, conforme as alterações detectadas. Após o início da atividade física, os pacientes são acompanhados por meio de consultas trimestrais e da repetição periódica dos exames citados.

Os pacientes são submetidos a uma avaliação médica que consta de anamnese e exame físico, visando à busca dos fatores de RCV. Os pacientes foram questionados sobre: história prévia pessoal e familiar de DCV, DM2 e dislipidemias. Exame físico completo foi realizado, dando ênfase aos níveis de pressão arterial e às medias antropométricas. A pressão arterial foi aferida no braço direito, com o paciente sentado, com esfigmomanômetro analógico, em duas aferições, nas condições minimamente ideais - paciente sentado, sem presença de esforço físico, vontade de urinar ou defecar, dor, ansiedade, ingestão de café ou uso de tabaco ou drogas ilícitas nos últimos minutos. Foi utilizado manguito adequado ao perímetro braquial do paciente. $\mathrm{O}$ peso foi medido em quilogramas, em balança analógica de plataforma, padrão hospitalar, com precisão de 100g. A altura foi medida em metros, com o paciente em pé, descalço sobre a plataforma da balança, com o olhar no horizonte. A circunferência abdominal (ou cintura) foi medida com o paciente em pé, no ponto médio entre a espinha ilíaca anterior e o último arco costal. A medida foi obtida em centímetros, com fita métrica de precisão de um milímetro.

Em seguida, cada paciente foi submetido aos seguintes exames complementares: níveis séricos de glicemia após 12 horas de jejum e após três dias de dieta livre; Teste Oral de Tolerância à Glicose (TOTG - glicemia após 2 horas com sobrecarga de $75 \mathrm{~g}$ de dextrose oral); perfil lipídico (colesterol total (CT), HDL-colesterol, triglicérides (TG) e LDL-colesterol calculado através da fórmula de Friedewald: LDL-colesterol $=$ CT-(HDL-colesterol + TG/ 5); hemograma completo, eletrólitos, uréia e creatinina. Foram excluídos do estudo os pacientes com avaliação clínica e laboratorial incompleta.

Para a definição dos fatores de risco foram utilizados os critérios descritos na tabela $1^{6,8,10,11,12}$. O RCV foi determinado pelo Escore de Framingham a partir dos seguintes fatores: idade, gênero, presença ou não de DM2 e tabagismo, níveis séricos de colesterol total, HDLcolesterol e pressão arterial. A cada um desses fatores foi atribuído um valor pré-estabelecido, depois somado aos demais. O resultado é um valor final que é correlacionado com uma tabela de riscos percentuais para o desenvolvimento de DAC em dez anos (tabela 1) ${ }^{4}$.

O diagnóstico de SM foi realizado pelos critérios 
Tabela 1. Critérios Diagnósticos para os Fatores de Risco Cardiovasculares

\begin{tabular}{|c|c|}
\hline Fatores de Risco & Critérios \\
\hline Sedentarismo & Ausência de atividade física regular nos últimos 30 dias \\
\hline Tabagismo & Consumo de qualquer número de cigarro nos últimos 30 dias \\
\hline Obesidade & $\begin{array}{l}\text { Índice de massa corpórea }-\mathrm{IMC}^{3} 30 \mathrm{~kg} / \mathrm{m}^{2} \text {, sendo classificado como obesidade grau I }(30 \geqslant \\
\mathrm{IMC}<35), \mathrm{II}(35 \geqslant \mathrm{IMC}<40) \text { e III }(\mathrm{IMC} \geqslant 40) . \\
\text { O sobrepeso foi definido como IMC } \geqslant 25 \mathrm{e}<30 .\end{array}$ \\
\hline Obesidade Abdominal & Circunferência abdominal (cintura) $\geqslant 102 \mathrm{~cm} \widehat{\jmath}$ e $88 \mathrm{~cm} q$ \\
\hline Diabetes mellitus & $\begin{array}{l}\text { Pelo menos dois resultados de glicemia de jejum }(\mathrm{G} j) \geqslant 126 \mathrm{mg} / \mathrm{dL} \text {, glicemia casual }>200 \\
\mathrm{mg} / \mathrm{dL} \text { na presença de sintomas clássicos (poliúria, polidipsia, polifagia e perda de peso) ou } \\
\text { glicemia após TOTG } \geqslant 200 \mathrm{mg} / \mathrm{dL} \text {. A Gj alterada foi definida como } 2 \text { medidas de } \mathrm{GJ} \geqslant 110 \mathrm{e} \\
<126 \mathrm{mg} / \mathrm{dL} \text {. A tolerância diminuída à glicose foi definida como glicemia após TOTG } \\
\geqslant 140 \mathrm{e}<200 \mathrm{mg} / \mathrm{dL}^{10,11} \text {. }\end{array}$ \\
\hline Dislipidemia & $\begin{array}{l}\text { Triglicerídeos } \geqslant 150 \mathrm{mg} / \mathrm{dL} ; \text { HDL-colesterol }<40 \mathrm{mg} / \mathrm{dL} \text { ठै }<50 \mathrm{mg} / \mathrm{dL} \uparrow \text {; Colesterol total } \\
>200 \mathrm{mg} / \mathrm{dL} ; \text { e LDL colesterol }>160 \mathrm{mg} / \mathrm{dL} \text {. Denominou-se dislipidemia mista a presença } \\
\text { de dois ou mais dos transtornos acima. }\end{array}$ \\
\hline
\end{tabular}

do National Cholesterol Education Program - Adult Treatment Panel (NCEP ATP III) e da I Diretriz Brasileira de Diagnóstico e Tratamento da Síndrome Metabólica (IDBSM ${ }^{6,8}$.Segundo esses critérios, considera-se SM a presença de pelo menos três dos seguintes componentes: obesidade abdominal (cintura $>102 \mathrm{~cm}$ nos homens e $88 \mathrm{~cm}$ nas mulheres), hipertrigliceridemia $(\geqslant 150 \mathrm{mg} / \mathrm{dL})$ ou baixos níveis de HDL-colesterol $(<40 \mathrm{mg} / \mathrm{dL}$ nos homens e 50 $\mathrm{mg} / \mathrm{dL}$ nas mulheres), hipertensão arterial (pressão arterial sistólica $\geqslant 130 \mathrm{mmHg}$ ou diastólica $\geqslant 85 \mathrm{mmHg}$ ) e finalmente alteração da homeostase da glicose (glicemia de jejum maior ou igual a $110 \mathrm{mg} / \mathrm{dL}$ ).

Os dados foram apresentados como média ( $\pm \mathrm{SD})$, mediana (variância) e/ou percentual. Foram utilizados o teste Qui-quadrado $\left(\chi^{2}\right)$ e o teste de Mann-Whitney/ Wilcoxon de comparação de amostras para averiguar a significância estatística das variáveis categóricas e numéricas, respectivamente.

\section{Resultados}

Foram avaliados 48 pacientes, com idades entre 23 e 74 anos, sendo sete homens (15\%) e 41 mulheres (85\%).

Dos pacientes, $67 \%$ tinham mais de 50 anos e $46 \%$, mais de 55. A idade média entre as mulheres foi de $54 \pm 9,7$ anos. Entre os homens, a média de idade foi de $60 \pm 13$ anos.

A prevalência global de tabagismo foi de 15\%, sendo que, dentre este percentual, $12 \%$ entre as mulheres e $29 \%$ entre os homens. A prevalência de tabagismo (29\%) foi semelhante aos dados da população masculina brasileira (34\%), porém observamos uma menor prevalência $(\mathrm{p}<0,05)$ entre as mulheres (12\% - IC 95\% 8,73-16,28), com relação aos dados disponíveis para mulheres brasileiras $(29 \%)^{13}$.

As prevalências dos fatores de risco na amostra estão descritas na tabela 2 e a comparação com os dados da literatura está descrita na tabela 3. A prevalência de sedentarismo foi de $52 \%$, sendo que, dentre este, $51 \%$ entre as mulheres e $57 \%$ entre os homens. Não houve diferença significativa na prevalência de sedentarismo, quando comparada com os dados disponíveis para a população adulta de uma região urbana brasileira ${ }^{14}$. A prevalência de história familiar positiva para DM2 e/ou DCV foi de 79\%, sendo $86 \%$ entre os homens e $78 \%$ entre as mulheres.

A prevalência de obesidade foi de 35\%, sendo $39 \%$ entre as mulheres (nove pacientes com grau I; duas com grau II e cinco com grau III) e 14\% entre os homens (um paciente, com obesidade grau II). Entre as mulheres, a prevalência foi superior $(\mathrm{p}<0,05)$ à da população brasileira, 
Tabela 2 - Projeto Atividade Física na Vila - Prevalências dos Fatores de Risco

\begin{tabular}{lccc}
\hline \multicolumn{1}{c}{ Fatores de Risco } & Mulheres $(\mathbf{n = 4 1 )}$ & Homens $(\mathbf{n}=\mathbf{7})$ & Total \\
\hline Tabagismo & 5 & 1 & 14,6 \\
Sedentarismo & 21 & 4 & 52,0 \\
História Familiar de DAC ou DM2 & 32 & 6 & 79,1 \\
Obesidade & 16 & 1 & 35,4 \\
Sobrepeso & 17 & 4 & 43,7 \\
Obesidade Abdominal & 29 & 1 & 60,4 \\
Hipercolesterolemia & 25 & 5 & 62,5 \\
HDL baixo & 4 & 1 & 10,4 \\
Hipertrigliceridemia isolada & 1 & 0 & 2,1 \\
Dislipidemia mista & 14 & 2 & 33,3 \\
Hipertensão arterial & 27 & 4 & 56,2 \\
Diabetes mellitus & 3 & 2 & 10,4 \\
Glicose de Jejum Alterada & 9 & 1 & 20,8
\end{tabular}

Tabela 3. Prevalências dos fatores de risco cardiovascular nos participantes do projeto Atividade Física na Vila e na população em geral

\begin{tabular}{lccc}
\hline \multicolumn{1}{c}{ Fatores de Risco } & Participantes do Projeto & População Geral & Valor de p \\
\hline \multirow{2}{*}{ Tabagismo } & Homens: $28,7 \%$ & Homens: $33,8 \%$ & $\mathrm{NS}$ \\
& Mulheres: $12,1 \%$ & Mulheres: $29,3 \%$ & $<0,05$ \\
Sedentarismo & $52 \%$ & $56 \%$ & $\mathrm{NS}$ \\
Obesidade & Homens: $14,3 \%$ & Homens: $8,9 \%$ & $\mathrm{NS}$ \\
& Mulheres: $39 \%$ & Mulheres: $13,1 \%$ & $\mathrm{p}<0,05$ \\
Sobrepeso & Homens: $57,1 \%$ & NS & $\mathrm{NS}$ \\
& Mulheres: $41,4 \%$ & Mulheres: $40 \%$ & $\mathrm{p}<0,05$ \\
Hipercolesterolemia & $62,5 \%$ & $40 \%$ & $\mathrm{p}<0,05$ \\
Hipertensão Arterial & $56,2 \%$ & $31,5 \%$ & $\mathrm{NS}$ \\
Diabete mellitus & $10,4 \%$ & $7,6 \%$ & $\mathrm{NS}$ \\
Glicose de Jejum Alterada & $20,8 \%$ & $8 \%$ & $\mathrm{NS}$ \\
Síndrome Metabólica & $27,1 \%$ & $23,7 \% *$ & \\
\hline
\end{tabular}

* Dados da população adulta norte-americana. Não há dados brasileiros até o momento.

conforme os dados disponíveis $(13 \%)^{15}$. A prevalência de sobrepeso foi de $44 \%$, sendo $57 \%$ entre os homens e $41 \%$ entre as mulheres, não havendo diferença estatisticamente significativa em relação aos dados populacionais brasileiros ${ }^{15}$. Somadas as prevalências de obesidade e sobrepeso, temos uma prevalência global de $79,2 \%$ de pacientes acima do peso ideal. A prevalência de obesidade abdominal foi de $60,4 \%$, sendo $14,3 \%$ entre os homens e $70,7 \%$ entre as mulheres. Não existem dados representativos sobre a obesidade abdominal na população brasileira.

A prevalência de hipertensão arterial foi de 56\% (IC $95 \% 70,8-41,7)$ - 57\% no sexo masculino e $56 \%$ no feminino, 
sendo significativamente maior do que a prevalência da população brasileira (15$32 \%)^{16,17,18,19,20}$.

A prevalência de diabetes mellitus foi de 10\% (IC 95\% 19,4-1,5) - $7 \%$ entre as mulheres e $29 \%$ entre os homens -, sem diferença em relação à população adulta brasileira $(7,6 \%)^{21}$. A prevalência de glicemia de jejum alterada foi de 21\% (IC 95\% 32,8-8,9), sendo $22 \%$ entre as mulheres e $14 \%$ entre os homens, semelhante aos dados da literatura brasileira.

A prevalência global de dislipidemia foi de $85 \%$, sendo $85 \%$ entre as mulheres e $86 \%$ entre os homens. Deste grupo, 60\% apresentaram aumento isolado de colesterol total, 47\% dislipidemia do tipo mista, 17\% baixo HDLColesterol e 13\% hipertrigliceridemia isolada. A prevalência total de hipercolesterolemia (63\% - IC 95\% 48,3-76,7) foi maior $(\mathrm{p}<0,05)$ do que na população adulta brasileira $(40 \%)$, conforme dados de Martinez e colaboradores ${ }^{22}$.

A prevalência de SM foi de $27 \%$ (IC 95\% 14,040,0) - 29\% nos homens e $27 \%$ nas mulheres -, sem diferença em relação à população adulta norte-americana $(24 \%)^{6,8,23}$. Ainda não há estimativas representativas da população brasileira.

O Escore de Framingham variou entre os valores de -11 a 16, com média de 5,2 \pm 5,5, sendo entre as mulheres

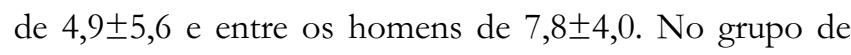
pacientes com SM, o escore de Framingham foi de 9,5 \pm 3,1 (IC 95\% 7,7-11,2), frente a um escore de 3,5 \pm 5,4 (IC $95 \% 1,6-5,4)$ no grupo sem SM $(p<0,05)$ (gráfico 1).

\section{Discussão}

Apesar da redução global na morbidade e mortalidade cardiovascular ao longo dos últimos 30 anos em países desenvolvidos, têm ocorrido elevações relativamente rápidas e substanciais em países em desenvolvimento, dentre os quais o Brasil. De acordo com as projeções da Organização Mundial de Saúde (OMS), esta tendência de elevação na DCV tende a persistir, agravando ainda mais o quadro de morbimortalidades elevadas nestes países ${ }^{24}$.

A doença coronariana tem origem multifatorial, portanto é importante considerar simultaneamente todos os riscos, na estimativa do risco individual. Tradicionalmente, as diretrizes de fatores de risco focam a avaliação de fatores únicos, como o controle da pressão arterial, dislipidemias e diabetes. Isso resulta em uma ênfase muito grande para elevações de valores em fatores isolados, em vez de uma visão do risco multifatorial ${ }^{25}$.

O envelhecimento da população tem visto um desvio do risco para a DCV em adultos mais idosos ${ }^{26}$. A idade desempenha um papel maior no risco absoluto de eventos coronarianos, aumentando também o impacto dos outros fatores sobre o risco de um indivíduo determinado ${ }^{25}$. Este é um referencial importante para iniciar a discussão dos resultados deste estudo, no qual 46\% dos pacientes tinham mais de 55 anos.

$\mathrm{Na}$ amostra estudada houve significativa preponderância do sexo feminino. Em relação a este fato vale lembrar o efeito protetor do sexo feminino, porém, podendo ser anulado pela presença de múltiplos fatores de risco ${ }^{25}$, como é o caso da maioria dos pacientes em questão.

A presença de história familiar precoce de DAC é definida pela IV Diretrizes Brasileiras sobre Dislipidemias e Prevenção de Aterosclerose (IVDBDPA) da Sociedade Brasileira de Cardiologia como infarto fatal ou não-fatal 
em parente de primeiro grau menor de 55 anos se masculino e menor que 65 anos se feminino ${ }^{24}$. Sabe-se da associação entre história familiar de DAC prematura e maior incidência nos descendentes. Entretanto, pelo fato de a hereditariedade ser um fator de risco imutável, nos indivíduos com história familiar, em termos práticos - e com o objetivo de reduzir o impacto desse fator sobre a morbidade e mortalidade cardiovasculares -, devemos controlar os fatores de risco que podem ser modificados, como: tabagismo, sedentarismo, obesidade, HA, DM2, dislipidemias, dentre outros.

As metanálises que avaliam a relação entre o grau de atividade física do indivíduo e a DCV demonstram aproximadamente o dobro de risco de doença nos inativos em comparação aos ativos. Dentre os possíveis mecanismos que poderiam estar envolvidos na cardioproteção exercida pela atividade física regular, estão os efeitos positivos sobre vários fatores de risco ${ }^{8,14,24}$. Assim, atuam beneficamente no controle de peso, na resistência à insulina, na pressão arterial, no perfil lipídico e na trombose. Em nosso estudo, observamos uma alta prevalência de sedentarismo em ambos os sexos, mas chama a atenção, entre as mulheres, a baixa prevalência do tabagismo, comparada aos dados brasileiros. Tal fato pode ser interpretado como a expressão de uma população motivada para a busca da saúde, mas que ainda não conseguiu implementar a atividade física como prática regular. A relação entre o fumo e o aumento do RCV está amplamente demonstrada em estudos anatomopatológicos e epidemiológicos. O risco de DAC é de duas a quatro vezes maior nos fumantes e diretamente proporcional ao número de cigarros consumidos ao dia ${ }^{8,13,24}$.

Observamos a alta prevalência de obesidade, principalmente quando considerado o sexo feminino, bem como a alta prevalência de obesidade abdominal, que é um dado fortemente relacionado à $\mathrm{SM}^{27}$. Vale lembrar que alguns grupos de trabalho já utilizam valores mais baixos da circunferência abdominal, que têm sido considerados mais apropriados a populações de diferentes etnias, como $94 \mathrm{~cm}$ para homens e $80 \mathrm{~cm}$ para mulheres ${ }^{28,29}$. Os valores aqui adotados foram escolhidos por serem oriundos de grandes diretrizes como o NCEP ATP III e a I-DBSM ${ }^{6,8}$. A população estudada apresentou um alto índice de $\mathrm{HA}^{16,17}$ 18, 19, 20, acima da média nacional, e de DM2 $2^{21}$, que, embora significativos, não diferiram estatisticamente dos dados populacionais brasileiros. Um dado que salta aos olhos é a presença de dislipidemia em quase todos os pacientes. A taxa de hipercolesterolemia total é superior à da população brasileira $^{22}$. O baixo HDL-colesterol, dado que muitas vezes passa despercebido, embora represente fator de RCV independente, também teve alta prevalência. Esta alta incidência de dislipidemias, muitas vezes com baixo HDLcolesterol isoladamente, está associada ao aumento da circunferência abdominal e ao sedentarismo, altamente prevalentes em nosso grupo. O HDL-colesterol baixo é um fator cujo tratamento mais efetivo é sem dúvida a atividade física regular ${ }^{2,4,9,27,30}$, o que novamente reforça a importância do projeto Atividade Física na Vila.

$\mathrm{Na}$ prática, os médicos lidam com um indivíduo complexo, e não com componentes particulares de sua vida. Determinados grupos de fatores de risco têm efeito multiplicativo, e um indivíduo com um número modesto de fatores pode apresentar um risco consideravelmente maior que uma pessoa com um único fator de risco importante. ${ }^{25}$ Percebe-se nos resultados deste trabalho, em acordo com a literatura atual ${ }^{2,4,9,25}$, o claro sinergismo entre os fatores de risco, evidenciado pelo maior escore de Framingham entre os pacientes com SM, o que denota maior risco cardiovascular global. O termo "fator de risco" apareceu pela primeira vez no título de um artigo médico em $1963^{5}$. Desde então, intensificou-se a busca por novos fatores de risco, tornando-se atualmente um modismo. Entretanto, apenas recentemente a literatura buscou estudar a relação de interdependência entre tais fatores, trazendo resultados esclarecedores.

O conceito de Síndrome Metabólica vem contribuir para este entendimento, não como uma nova "doença da moda", mas chamando a atenção para a correlação entre estilo de vida, obesidade abdominal e RCV global. Como um conceito que denota o agrupamento de fatores de risco, a SM tem sido um paradigma útil. Isto é, chama atenção para o fato de que alguns fatores de risco para o desenvolvimento 
de DCV têm efeito multiplicativo e se agrupam em pacientes predispostos. O ponto pedagógico implicado pelo termo é que a identificação de uma das variáveis de risco deve incitar a busca para outros ${ }^{31}$. Este entendimento é relativamente inovador, na medida em que consideramos que o escore de Framingham não leva em conta alguns componentes da SM, em especial a obesidade abdominal. Segundo a IVDBDPA, em qualquer categoria de risco cardiovascular, a presença da SM constitui um fator agravante ${ }^{24}$.

Além disso, devemos reconhecer a natureza progressiva da SM. Na história natural da SM, o paciente primeiro adquire obesidade abdominal sem fatores de risco, mas, com o tempo, múltiplos fatores de risco começam a aparecer. Quando a diabetes não está presente, o risco de progressão para DM2 é cinco vezes maior quando comparado com aqueles sem SM. Em alguns pacientes, a síndrome culmina em DM2 e o risco para DCV aumenta ainda mais. Quando a DCV se desenvolve, complicações cardiovasculares como arritmias, falência cardíaca e episódios trombóticos freqüentemente aparecem. Aqueles com DM2 podem posteriormente adquirir várias complicações incluindo cardiomiopatia diabética, várias neuropatias e falência renal. Quando o DM2 e a DCV existem concomitantemente, o risco para subseqüente morbidade cardiovascular é muito alto ${ }^{32}$.

A importância dos fatores de risco metabólico, tanto pela perspectiva clínica quanto da saúde pública, é que a prevenção, a detecção e a reversão podem ocorrer antes do desenvolvimento da DCV manifesta. Deve-se ressaltar que a Obesidade e, por conseqüência, a Síndrome Metabólica vêm aumentando paulatinamente sua incidência em toda as faixas etárias e que hábitos alimentares saudáveis e atividade física regular são ferramentas-chave na reversão dessa tendência ${ }^{4,5,9}$.

Cada vez mais, os dados nos levam ao entendimento da necessidade premente da mudança no estilo de vida para a redução da morbimortalidade cardiovascular dos pacientes, não apenas documentando isoladamente a presença de fatores de risco, mas objetivando principalmente uma abordagem global com condutas de promoção e proteção da saúde. É como se nos aproximássemos gradualmente daquela milenar sabedoria encerrada no conhecido provérbio chinês: "Buscar a saúde somente quando já se está doente é o mesmo que esperar a sede para começar a cavar um poço" 33 .

\section{Referências}

1.Brasil. Ministério da Saúde. Datasus. Mortalidade proporcional por causas selecionadas por região do Brasil, 1998. Disponível em: http:// www.datasus.gov.br. Acesso em: 14 fev. 2005.

2. Sowers JR, Epstein M, Frohlich ED. Diabetes, Hypertension, and Cardiovascular Disease, An Update. Hypertensio.n 2001; 37: 1053-9.

3. Braunwald E et al. Harrison Medicina Interna.15ed. Rio de Janeiro: [s.n]; 2002. p. 1462.

4. Peter W. F. et al. Prediction of Coronary Heart Disease Using Risk Factor Categories. Circulation. 1998; 97:1837-47. 5. Gordon T et al. Lipoproteins, cardiovascular disease and death. The Framingham study. Arch Intern Med. 1981; 141: 1128-31.

6. Third Report of the National Cholesterol Education Program (NCEP) Expert Panel on Detection, Evaluation and Treatment of High Blood Cholesterol in Adults (Adult Treatment Panel III). Final report. Circulation. 2002; 106:3143-421.

7. Teixeira RJ, Simão Y, Silva TT, Barroso S, Barbosa JSO. Síndrome Metabólica: Diagnóstico, Prevenção e Tratamento. In: Curso Pré-congresso do $43^{\circ}$. Congresso Científico do Hospital Universitário Pedro Ernesto. Rio de Janeiro, agosto de 2005.

8. I Diretriz Brasileira de Diagnóstico e Tratamento da Síndrome Metabólica. Arq Bras Card. 2005; 84:1-28.

9. Lindsay RS, Howard BV. Riscos Cardiovasculares Associados com a Síndrome Metabólica. Current Diabetes Reports-Latin America. 2004, 3: 267-72.

10. Alberti KG, Zimmet PZ: Definition, diagnosis and classification of diabetes mellitus and its complications. Part 1: diagnosis and classification of diabetes mellitus provisional report of a WHO consultation. Diabet Méd. 
1999, 15: 539-53.

11. American Diabetes Association. Padronização de Cuidados Médicos em Diabetes. Diabetes Care. 2004; 3: 64-95.

12. IV Diretrizes Brasileira de Hipertensão Arterial. Arq Bras Cardiol. 2004; 82:1-40.

13. Achutti AC, Rosito MHE, Achutti VAR. Tabagismo. In: Medicina Ambulatorial: Condutas de Atenção Primária Baseada em Evidências. Bruce B. Duncan e cols. Porto Alegre (RS): Artmed; 2004. p. 533.

14. Rego A et al. Fatores de risco para doenças crôniconão-transmissíveis: inquérito domiciliar no município de São Paulo, SP (Brasil). Metodologia e resultados preliminares. Rev Saúde Pública. 1990; 24:277-85.

15. Instituto Brasileiro de Geografia e Estatística (IBGE). Pesquisa de orçamentos familiares, período 2002-2003. Tabela 1: Prevalência de déficit de peso, excesso de peso e obesidade na população com 20 anos de idade ou mais, por sexo, segundo Unidade da Federação, áreas urbanas dos Municípios das Capitais e Regiões Metropolitanas. Disponível em: www.ibge.gov.br/home/estatistica/ populacao. Acesso em: 07 abr. 2006.

16. Gus I, Fischmann A, Medina C. Prevalência dos fatores de risco da doença arterial coronariana no Estado do Rio Grande do Sul. Arq Bras Cardiol. 2002; 78: 478-83.

17. Lolio Ca, Pereira JCR, Lotufo PA, Souza JMP. Hipertensão Arterial e Possíveis Fatores de Risco. Rev Saúde Pública. 1993; 27: 357-62.

18. Piccini RX, Victora CG. Hipertensão Arterial Sistêmica em Área Urbana no Sul do Brasil. Rev Saúde Pública. 1994; 28: 261-7.

19. Bloch KV, Klein CH, Silva NAS, Nogueira AR, Campos LHS. Hipertensão Arterial e Obesidade na Ilha do Governador - Rio de Janeiro. Arq Bras Cardiol. 1994; 63: 17-22.

20. Avezum A, Guimarães HP, Berwanger O, Piegas LS. Hipertensão Arterial e Risco Cardiovascular. In: Tópicos Especiais em Hipertensão Arterial. Emílio Antonio Francischetti e Antonio Felipe Sanjuliani (orgs.). São Paulo: Libbs Farmacêutica; 2005. p. 16.
21. Estudo Multicêntrico Sobre a Prevalência do Diabetes Mellitus no Brasil. 1988. Disponível em:

h t t p : / / w w w.diabetes.org.br/suces sos / apresentacao_estudomulticentrico.php. Acesso em: 08 abr. 2006.

22. Martinez et al. Determinação do nível de colesterol elevado em brasileiros. Arq Bras Cardiol. 2003; 80: 631-4. 23. Ford ES, Giles, MSC, Dietz WH. Prevalence of the metabolic syndrome among US adults: findings from the Third National Health and Nutritional Examination Survey. JAMA. 2002; 287: 356-9.

24. Sociedade Brasileira de Cardiologia. IV Diretrizes Brasileira sobre Dislipidemias e Prevenção da Aterosclerose Departamento de Aterosclerose da Sociedade Brasileira de Cardiologia. Arq Bras Cardiol. 2007; 88: 2-19.

25. Saraiva FJK. Como Estratificar o Risco Cardiovascular. In: Manual de Dislipidemias e Cardiometabolismo. São Paulo: Farmalab; 2004. p 55-65.

26. Vita AJ et al. Aging, health risk, and cumulative disability. N Engl J Med. 1998; 338:1035-41.

27. Palaniappan L et al. Predictors of the Incident Metabolic Syndrome in Adults. Diabetes Care. 2004; 27: 788-93.

28. Lean MEJ, Han TS, Morrison CE. Waist circunferência as a measure for indicating the Neide for weight management. BMJ. 1995; 311: 158-61.

29. Gang H, Qiao Q, Tuomilehto J, Balkau B, Borch-Johnsen K, Pyorala K for the DECODE Study Group. Prevalence of the metabolic syndrome and its relatem to al-cause and cardiovascular mortality in nondiabetic European men and women. Arch Intern Méd. 2004; 164: 1066-76.

30. Teixeira RJ, Silva TT, Barbosa JSO, Salek M, Cavaliere MLA, Souza JMA, Rodrigues RD. Prevenindo a Síndrome Plurimetabólica Através da Prática de Atividade Física. In: Apresentação do Projeto "Atividade Física na Vila". Disciplina de Medicina Integral e Disciplina de Educação Física. Hospital Universitário Pedro Ernesto e Pavilhão João Lira Filho da Universidade do Estado do Rio de Janeiro. Rio de Janeiro, 15 de outubro de 2004.

31. Declaração Conjunta da Associação Americana de Diabetes e da Associação Européia para o Estudo do 
Diabetes. Diabetes Care. 2005; 28: 2289-304. [Edição em português].

32. Grundy SM. Metabolic Syndrome: Connecting and reconciling Cardiovascular and Diabetes Worlds. JACC. 2006;

47: 1093-100.

33.Capra, F. O Ponto de Mutação. São Paulo: Cultrix; 1985.

\section{Endereço para correspondência}

Rosimere de Jesus Teixeira

Rua General Espírito Santo Cardoso, 377 apt 403

Rio de Janeiro/RJ.

CEP: 20.530 .500

\section{Endereço eletrônico}

rosijt@fst.com.br 